\title{
Distinct Patterns of Temporal and Directional Connectivity among Intrinsic Networks in the Human Brain
}

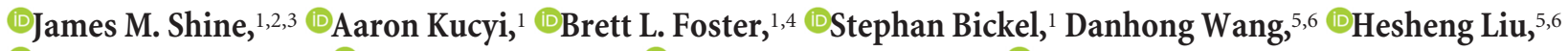

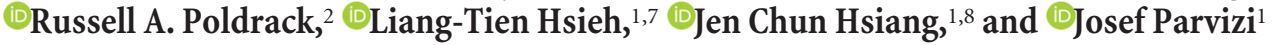 \\ ${ }^{1}$ Laboratory of Behavioral and Cognitive Neuroscience, Stanford Human Intracranial Cognitive Electrophysiology Program, Department of Neurology and \\ Neurological Sciences, Stanford University, Stanford, California 94301, 2Department of Psychology, Stanford University, Stanford, California 94301, \\ ${ }^{3}$ Central Clinical School, The University of Sydney, Sydney, 2050 New South Wales, Australia, ${ }^{4}$ Department of Neurosurgery, Baylor College of Medicine, \\ Texas 77030, ${ }^{5}$ Athinoula A. Martinos Center for Biomedical Imaging, Department of Radiology, Massachusetts General Hospital, Harvard Medical School, \\ Charlestown, Massachusetts 02114, ${ }^{6}$ Department of Psychiatry, Massachusetts General Hospital, Harvard Medical School, Boston, Massachusetts 02114, \\ ${ }^{7}$ Center for Neuroscience, University of California, Davis, California 63110, and ${ }^{8}$ Neuroscience PhD Program, Washington University, St. Louis, Missouri 63130
}

To determine the spatiotemporal relationships among intrinsic networks of the human brain, we recruited seven neurosurgical patients (four males and three females) who were implanted with intracranial depth electrodes. We first identified canonical resting-state networks at the individual subject level using an iterative matching procedure on each subject's resting-state fMRI data. We then introduced single electrical pulses to fMRI pre-identified nodes of the default network (DN), frontoparietal network (FPN), and salience network (SN) while recording evoked responses in other recording sites within the same networks. We found bidirectional signal flow across the three networks, albeit with distinct patterns of evoked responses within different time windows. We used a data-driven clustering approach to show that stimulation of the FPN and SN evoked a rapid $(<70 \mathrm{~ms})$ response that was predominantly higher within the SN sites, whereas stimulation of the DN led to sustained responses in later time windows $(85-200 \mathrm{~ms})$. Stimulations in the medial temporal lobe components of the DN evoked relatively late effects ( $>130 \mathrm{~ms}$ ) in other nodes of the DN, as well as FPN and SN. Our results provide temporal information about the patterns of signal flow between intrinsic networks that provide insights into the spatiotemporal dynamics that are likely to constrain the architecture of the brain networks supporting human cognition and behavior.

Key words: default network; electrical evoked potentials; frontoparietal network; intracranial EEG; salience network

Significance Statement

Despite great progress in the functional neuroimaging of the human brain, we still do not know the precise set of rules that define the patterns of temporal organization between large-scale networks of the brain. In this study, we stimulated and then recorded electrical evoked potentials within and between three large-scale networks of the brain, the default network (DN), frontoparietal network (FPN), and salience network (SN), in seven subjects undergoing invasive neurosurgery. Using a data-driven clustering approach, we observed distinct temporal and directional patterns between the three networks, with FPN and SN activity predominant in early windows and DN stimulation affecting the network in later windows. These results provide important temporal information about the interactions between brain networks supporting human cognition and behavior.

\section{Introduction}

Recent evidence suggests that the time course of spontaneous hemodynamic fluctuations at rest characterizes an intrinsic func-

\footnotetext{
Received June 6, 2017; revised Aug. 21, 2017; accepted Aug. 25, 2017.

Author contributions: B.L.F., H.L., R.A.P., and J.P. designed research; B.L.F., S.B., L.-T.H., and J.P. performed research; J.P. contributed unpublished reagents/analytic tools; J.M.S., A.K., B.L.F., S.B., D.W., H.L., L.-T.H., and J.C.H. analyzed data; J.M.S. and J.P. wrote the paper.

The authors declare no competing financial interests.

Correspondence should be addressed to Dr. Josef Parvizi, Laboratory of Behavioral and Cognitive Neuroscience, Stanford Human Intracranial Cognitive Electrophysiology Program, Department of Neurology and Neurological Sciences, Stanford University, 300 Pasteur Drive, Palo Alto, CA 94301. E-mail: parvizi@stanford.edu.
}

tional network architecture in the human brain that changes across multiple unique brain states (Buckner et al., 2013). However, neuroimaging methodologies such as fMRI are limited in their ability to delineate the fine-grained directional and temporal relationships within and between brain networks. Given these methodological limitations, it has remained largely unknown whether the relationships between intrinsic networks are causally symmetric and if the temporal patterns of signal propagation

DOI:10.1523/JNEUROSCI.1574-17.2017

Copyright $\odot 2017$ the authors $\quad 0270-6474 / 17 / 379667-08 \$ 15.00 / 0$ 
a

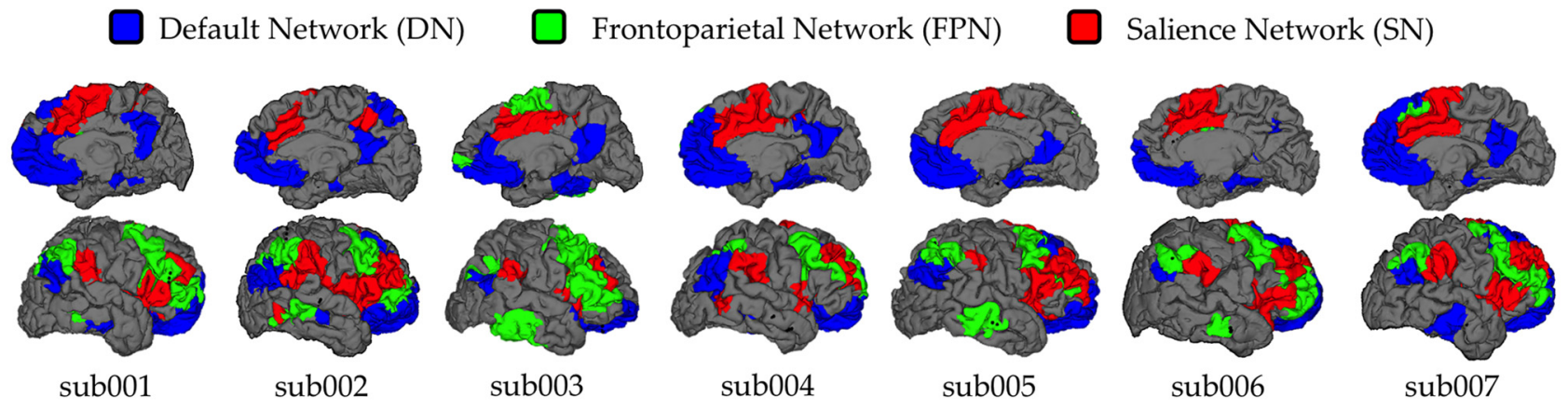

b

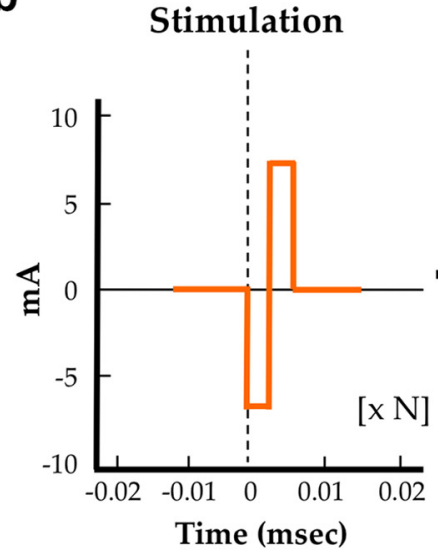

Evoked Response

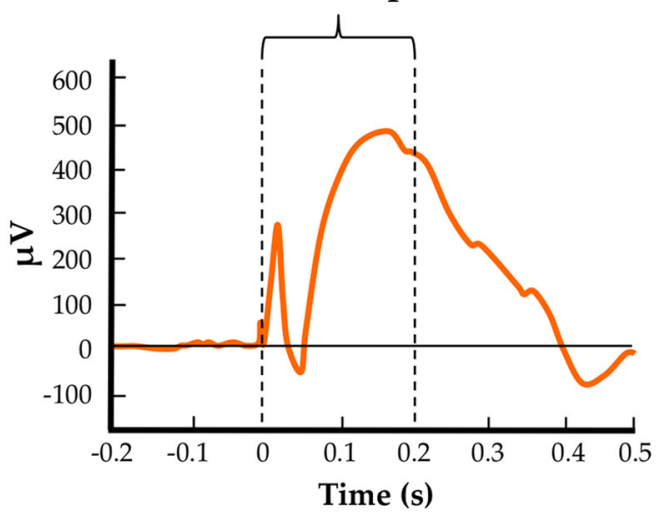

Figure 1. Subject-level network coverage and evoked responses. $\boldsymbol{a}$, Each of the three intrinsic networks studied (DN, blue; FPN, green; and SN, red) warped onto individual subject brains. Note the substantial variance of spatial coverage and extent for each of the three networks. $\boldsymbol{b}$, Pairs of electrodes were stimulated and evoked potentials were recorded from each other pair of electrodes. Shown is an example of a bipolar stimulation from a depth electrode depicting the typical deviation from baseline activity ( $\mu V$; $y$-axis) over time (ms; $x$-axis). Note that, due to directional ambiguity associated with bipolar recordings, we analyzed the absolute value of power from a bipolar montage.

within and between networks are equivalent. Improved clarity in this area will provide crucial insights into the functional relationships between intrinsic networks of the brain and thus will inform putative communication pathways between these networks.

We combined individual resting-state fMRI, intracranial recordings, and direct electrical stimulation of the cerebral cortex in neurosurgical subjects implanted with depth electrodes. The measurement of cortical evoked responses to distal cortical stimulation (often termed corticocortical evoked potentials; Fig. 1b) is a well established technique in clinical neurophysiology that is used to determine effective connections between two cortical sites of interest (Gollo et al., 2017). Although earlier responses are interpreted as emerging from direct synaptic connections, delayed and more jittered responses correlate with low-pass filtering of efferent signals via multisynaptic connections or through local circuits, thus implying connections via relatively indirect pathways (Keller et al., 2014).

Despite the significant benefits in signal-to-noise ratio offered by intracranial EEG recordings (Keller et al., 2014), clinical considerations limit the spatial coverage of electrodes. Therefore, it can be difficult to collect matching recording montages across subjects, particularly given the known idiosyncrasies associated with the functional organization of the brain (Laumann et al., 2015). In our study, we used two factors to mitigate this potential issue. The first involved the individualized assignment of electrodes to fMRI defined resting-state networks using a novel iterative procedure that tunes predefined network signatures to individual differences in resting-state connectivity (Fig. 1).
Note that the spatial pattern associated with individual network signatures demonstrates substantial heterogeneity across the subjects involved in this study (Fig. 1a), which is to be expected given the known presence of substantial differences in the resting brain across individuals (Gordon et al., 2017). The second approach involved the data-driven clustering of evoked time series (Fig. 2). Briefly, we identified clusters of similar evoked activity patterns without any prior knowledge of which networks were stimulated and recorded from. By applying a nonparametric permutation analysis to these data, we were then able identify the patterns of internetwork and intranetwork connectivity that were present in each cluster above chance (Fig. 3). Overall, the use of unsupervised, data-driven analytical approaches limited these constraints on the data and thus allowed us to infer spatiotemporal constraints over network-level interaction in the human brain.

Using this individualized multimodal approach, we were able to decipher the pattern of cortical evoked responses that reflect patterns of causal connectivity within and between three major intrinsic networks of the human brain (Seeley et al., 2007; Raichle, 2015): the default network (DN), which is associated with passive states of unconstrained cognition (Buckner et al., 2008); the cingulo-opercular "salience" network (SN), which is associated with stimulus orienting and task switching (Uddin, 2015); and the frontoparietal network (FPN), which is associated with cognitive control and goal-directed attention (Corbetta and Shulman, 2002). Our results provide insights into the spatiotemporal dynamics that are likely to constrain the architecture of the brain networks supporting human cognition and behavior. 
a

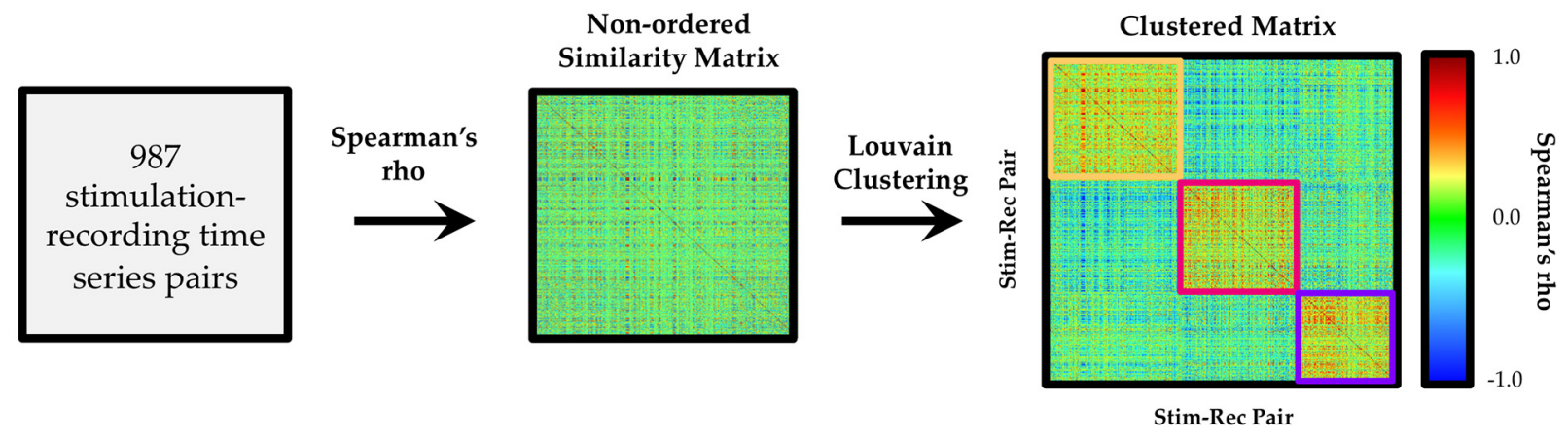

b

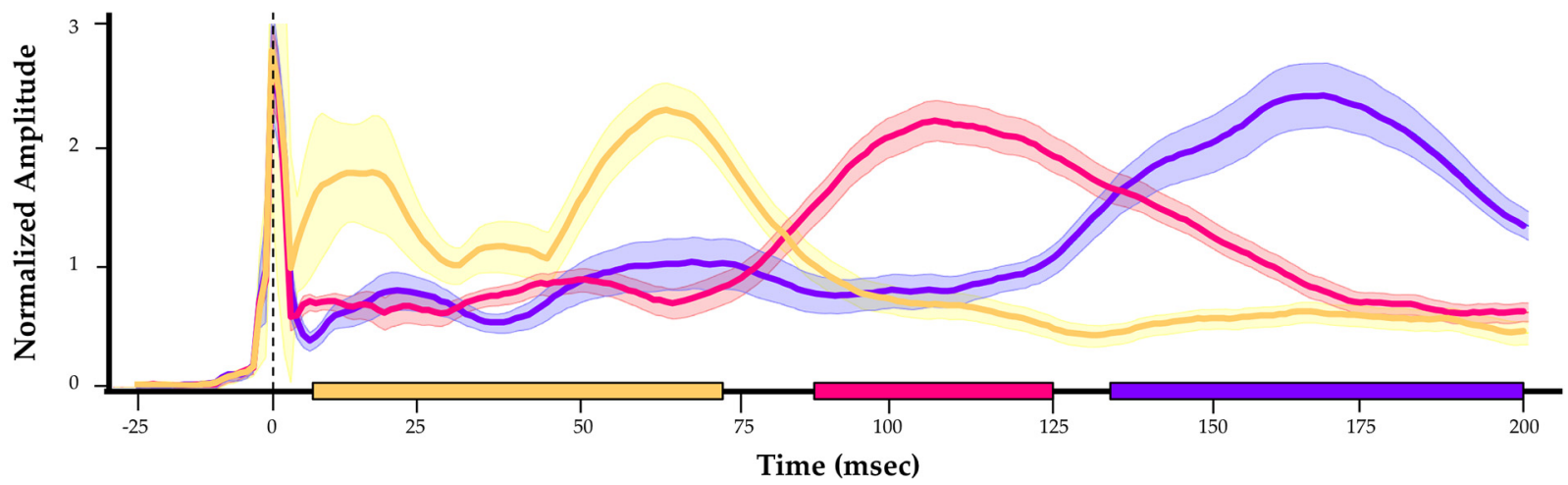

Figure 2. Time series clustering. $\boldsymbol{a}$, Raw time series from all 987 stimulation-recording pairs were temporally correlated (using a Spearman's $\rho$ ) across all stimulations in the seven subjects. The $987 \times 987$ similarity matrix was then clustered using the Louvain algorithm, which identified three distinct clusters in the data (denoted by orange, pink, and purple boxes). $\boldsymbol{b}$, The three clusters each demonstrated a unique temporal profile $( \pm \mathrm{SE}$ ) in the evoked responses across the seven subjects. Colors match the clusters identified using the Louvain algorithm: orange, $+11 \mathrm{to}+69 \mathrm{~ms}$; pink, +82 to $+125 \mathrm{~ms}$; and purple, +132 to $+200 \mathrm{~ms}$. Colored boxes on the $x$-axis denote time points that were associated with significantly elevated evoked activity in each of the three temporal clusters.

\section{Materials and Methods}

Subject details. Seven subjects ( $39.57 \pm 13.8$ years of age; $57 \%$ males) with focal epilepsy were recruited in this study. Each subject underwent stereotactic implantation of depth intracranial electrodes at Stanford Medical Center to localize the sources of their seizures. Only depth electrodes were used in this experiment. The location of electrode placement was determined solely by clinical needs (Fig. 1, Table 1). All participants gave written informed consent approved by the Stanford University Internal Review Board before their participation.

Electrical stimulation. Single pulse stimulations were performed with a bipolar setup in which single pulses of electrical current $(4-10 \mathrm{~mA}$, biphasic, $500 \mu \mathrm{s} /$ phase) were injected between pairs of intracranial electrodes using a cortical stimulator (Grass Technologies Model S12X) while subjects were awake and resting quietly. Electrical stimulation was delivered at $0.5 \mathrm{~Hz}$ ( 1 patient received $2 \mathrm{~Hz}$ stimulation), the magnitude of which varied between patients $(4-10 \mathrm{~mA})$ and was chosen so not to induce inadvertent epileptic discharges. Each electrode contained 10-14 contacts, through which electrical activity of the brain was recorded. Electrodes used in this study contained contacts that were cylinder shaped with a $0.86-1.10 \mathrm{~mm}$ circumference diameter and $2.29-2.41 \mathrm{~mm}$ height. The distance between the centers of two adjacent electrode contacts was $4-5 \mathrm{~mm}$. The total surface area of the electrode contacts ranged between 1 and $15 \mathrm{~mm}^{2}$. The direction of the dipoles recorded from the electrode contacts was variable depending on the plane of the electrode trajectory. Stimulations were performed with alternating polarity between the pair of stimulated electrodes; that is, the cathode and anode changed with each delivered single pulse. Due to the variability of testing time allowed for individual patients, the number of electrical stimulation trials varied between participants and differed across electrode pairs (mean: 48; range: 11-90 trials). The end result is a collection of time series, each of which is associated with stimulation and recording from a particular set of brain regions. Importantly, regions within white matter or ventricular space were identified using FreeSurfer's segmentation algorithm and removed before further analysis.

Controlling for volume conduction effects. To minimize volume conduction effects, we discarded data collected from the recording sites adjacent to the stimulated sites (i.e., on the same recording electrode shaft; $n=$ 209 pairs).

Analysis of evoked responses. Analysis of broadband electrophysiological data focused on evoked responses in nonstimulated electrodes. After bipolar re-referencing, evoked responses in nonstimulated electrodes were characterized by segmenting continuous EEG data into $225 \mathrm{~ms}$ epochs ( $25 \mathrm{~ms}$ prestimulation to $200 \mathrm{~ms}$ poststimulation; $1000 \mathrm{~Hz}$ sampling rate), which were time locked to the delivery of stimulation pulses (i.e., $t=0$ ). All electrode pairs identified as lying outside of gray matter were discarded, as were electrode pairs on the particular electrode shaft being stimulated in each experimental trial. Epoched data then underwent a rejection procedure in which evoked responses exceeding $\pm 200 \mu \mathrm{V}$ were excluded because these epochs may be contaminated by electrical artifacts. Time series data were normalized to the mean and SD of the voltage present within the $20 \mathrm{~ms}$ prestimulus window $(t=-25$ to -5$)$ for each individual subject, after which time the data were collated at the group level. It should be noted that we discounted the $5 \mathrm{~ms}$ immediately before each stimulation trial and $10 \mathrm{~ms}$ immediately after the stimulations to ensure that peristimulus artifacts did not represent a potential confound. Each time series was then inspected visually and data with abnormal activity in the prestimulus window (defined as $Z$-score $>3$ ) were discarded. Note that, although evoked responses consisted of both positive and negative voltage deflections, for the purposes of this study, we chose not to differentiate between the two polarities because the direction of activity is ambiguous in data collected from bipolar electrodes (Keller et al., 2014). Therefore, the data were collectively considered (by taking the absolute voltage deflection) to determine effective connectivity between brain regions. 
a

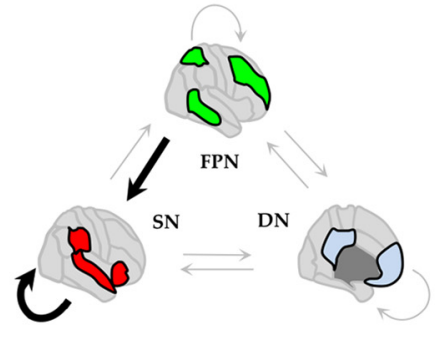

b

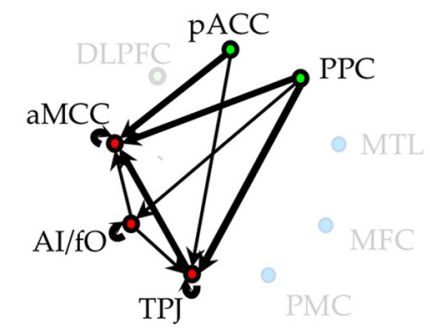

Cluster $1 \square$

\section{Networks}

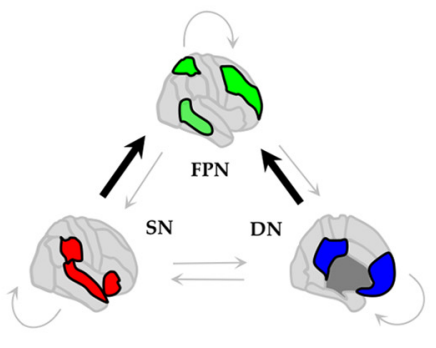

Regions

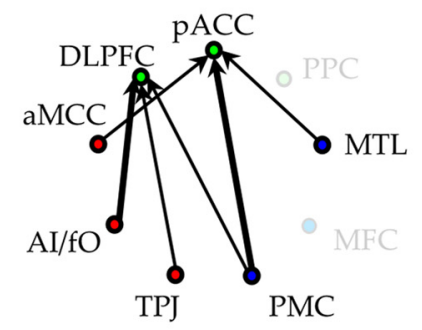

Cluster $2 \square$
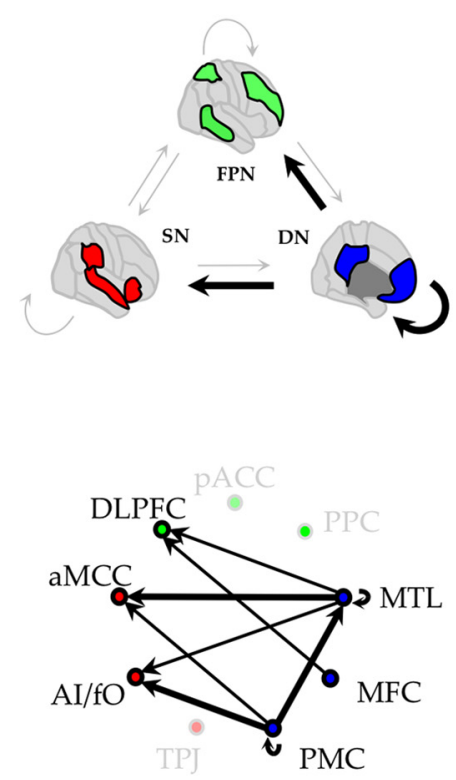

\section{Early}

Late

Figure 3. Network and regional signatures of evoked responses. $\boldsymbol{a}$, Graphical depiction of the significant activity within each cluster. Thick arrows designate the presence of a particular connection above chance levels. Note that the absence of a significant effect does not preclude the presence of a connection. $\boldsymbol{b}$, Regional patterns of effective connectivity within each cluster. Arrows depict significant effective connectivity (results of nonparametric permutation test: thick lines: $p<0.001$; thin lines: $p<0.05$ ). DLPFC, Dorsolateral prefrontal cortex.

Table 1. Network location of stimulated and recorded electrodes across all seven subjects

\begin{tabular}{lllllllll}
\hline \multicolumn{7}{c}{ Subject no. } & \multicolumn{1}{l}{ notal } \\
\cline { 2 - 8 } & 1 & 2 & 3 & 4 & 5 & 6 & 7 & Total \\
\hline Age & 43 & 41 & 28 & 25 & 63 & 50 & 29 & \\
Sex & F & M & F & M & F & M & M & \\
SN & $4(3)$ & $3(1)$ & $2(1)$ & $9(3)$ & $1(1)$ & $1(1)$ & $1(1)$ & $21(11)$ \\
aMCC & $1(1)$ & - & $2(1)$ & 2 & $1(1)$ & $1(1)$ & - & $7(4)$ \\
Al/f0 & - & $1(1)$ & - & $6(3)$ & - & - & $1(1)$ & $8(5)$ \\
TPJ & $3(2)$ & 2 & - & 1 & - & - & - & $6(2)$ \\
& & & & & & & & \\
FPN & $3(1)$ & $20(2)$ & $7(1)$ & $30(5)$ & $9(1)$ & $7(1)$ & $4(2)$ & $80(13)$ \\
DLPFC & 1 & $8(1)$ & - & $12(2)$ & 2 & - & $2(1)$ & $25(4)$ \\
pACC & $2(1)$ & $8(1)$ & $3(1)$ & $7(2)$ & $4(1)$ & - & $2(1)$ & $26(7)$ \\
PPC & - & 4 & 4 & $11(1)$ & 3 & $7(1)$ & - & $29(2)$ \\
DN & $14(2)$ & $17(2)$ & $9(1)$ & $22(2)$ & $24(1)$ & $8(2)$ & $25(2)$ & $119(12)$ \\
MTL & $9(1)$ & 13 & $9(1)$ & $9(1)$ & 13 & 3 & 16 & $72(3)$ \\
MFC & 4 & $1(1)$ & - & 12 & $11(1)$ & $1(1)$ & $9(2)$ & $38(5)$ \\
PMC & $1(1)$ & $3(1)$ & - & $1(1)$ & - & $4(1)$ & - & $9(4)$ \\
TOTAL & $21(6)$ & $40(5)$ & $18(3)$ & $61(10)$ & $34(3)$ & $16(4)$ & $30(5)$ & $220(36)$ \\
\hline
\end{tabular}

The value in each cell denotes the number of electrode pairs recorded from within each network and the number in parentheses within each cell denotes the number of electrode pairs stimulated within each network.

$\mathrm{Al} / \mathrm{FO}$, anterior insula/frontal operculum; aMCC, anterior medial cingulate cortex; DLPFC, dorsolateral prefrontal cortex; DN, default network; FPN, frontoparietal network; MTL, medial temporal lobe; MFC, medial frontal cortex; PMC, posteriomedial cortex; $\mathrm{PACC}$, prefrontal anterior cingulate cortex; PPC, posterior parietal cortex; $\mathrm{SN}$, salience network; TPJ, temporoparietal junction.

Resting-state fMRI acquisition and preprocessing. Resting-state fMRI data were obtained at the Center for Cognitive and Neurobiological Imaging and the Richard M. Lucas Center for Imaging at Stanford University. fMRI data for S1, S3, and S4 were acquired on a $3 \mathrm{~T}$ GE scanner using a 32-channel head coil ( 30 slices, $4.0 \mathrm{~mm}$ isotropic voxels, $\mathrm{TR}=2000 \mathrm{~ms}$, volumes $=180, \mathrm{FOV}=100 \mathrm{~mm}, \mathrm{TE}=30 \mathrm{~ms}$, flip angle $=77 \mathrm{deg}$, bandwidth $=127.68 \mathrm{kHz}$ ). fMRI data for S2, S5, S6, and S7 were obtained on a $3 \mathrm{~T}$ GE scanner using a spiral sequence with a 32-channel head coil (30 slices, $4.0 \mathrm{~mm}$ isotropic voxels, $\mathrm{TR}=2000 \mathrm{~ms}$, volumes: 240 , FOV $=$ $220 \mathrm{~mm}, \mathrm{TE}=30 \mathrm{~ms}$, flip angle $=77 \mathrm{deg}$, bandwidth $=127.68 \mathrm{kHz}$ ) (Glover and Law, 2001). Data were preprocessed using functions in the FMRIB Software Library (FSL version 5.0.8), with standard steps including slice-timing correction, motion correction, regression of nuisance parameters (head motion, whole-brain signal, ventricular and white matter time series), spatial smoothing with a $5 \mathrm{~mm}$ FWHM Gaussian kernel, and high-pass filtering at $0.01 \mathrm{~Hz}$. Note that data were not lowpass filtered to enhance the signal-to-noise ratio.

Network membership assignment. To determine the network membership associated with individual electrodes in individual participants, resting-state data were projected onto cortical surfaces using FreeSurfer and the vertices were clustered into a set of predefined networks using an iterative cortical parcellation approach (Wang et al., 2015). Specifically, a group-level atlas consisting of 17 networks (the "Yeo 17" atlas) was used as the initialization of the parcellation procedure (Yeo et al., 2011). Vertex-wise time courses were averaged across the vertices that fell within each network, resulting in 18 reference signals. The original fMRI signal at each vertex was then correlated to the 18 reference signals and each vertex was reassigned to the network with the maximal correlation to the reference signals. The ratio between the largest and the second largest correlation values was used as a confidence signal and all vertices with a confidence score $>1.1$ were averaged and termed the "core signal." For each network, the core signal and the original reference signals were averaged in a weighted manner (e.g., by multiplying the signal by the number of iterations and the signal-to-noise ratio). The resulting signal estimate was used as the new reference signal for the next iteration. The process was repeated through a number of iterations and the weights were gradually reduced over time. The procedure was stopped once net- 
work membership remained the same for $98 \%$ of the vertices in two consecutive iterations. Each electrode pair was then associated with the network identity that most closely matched its spatial location $(94.6 \%$ of the identified electrode pairs fell within the same network). DN nodes comprised networks 10, 15, and 16 from the Yeo 17 atlas (Yeo et al., 2011); FPN comprised networks 12 and 13; and SN comprised networks 7 and 8 (electrodes belonging to one of the other 10 networks were discarded). Due to our interest in the networks underlying cognition and attention, electrodes present within other resting-state networks were discarded before the present analyses $(N=182$ across seven subjects; numbers not included in final 987 time series).

Time series similarity and clustering analysis. Poststimulus time series were grouped according to the networks stimulated and recorded from and grouped together independently of individual subjects. Because the distribution of the time series was distinctly non-Gaussian (KolmogorovSmirnov test: $p<0.001$ ), we computed a Spearman's $\rho$ correlation between the poststimulus epoch $(t=+11$ to +200$)$ of each of the time series, which were each averaged across multiple $(\sim 40)$ stimulations of the same electrode pair. To determine the presence of data-driven clusters in our recorded intracranial EEG signals, we applied a weighted and signed version of the Louvain algorithm to the time series similarity matrix (Rubinov and Sporns, 2010). This procedure resulted in a clustering assignment for each pair of electrodes (i.e., both stimulated and recorded from in an individual subject). Importantly, we chose the Spearman's $\rho$ due to its utility in comparing non-Gaussian time series (all 987 time series were significantly non-normal using a one-sample Kolmogorov-Smirnov test) rather than for its ability to relate to a particular neurophysiological interpretation (for which other methods would be clearly more useful). Due to the stochastic nature of the algorithm, the process was repeated 500 times and a consensus clustering was obtained. This approach identified three distinct clusters $(\mathrm{Q}=0.42 \pm$ 0.1 ), each of which was evenly distributed across the seven subjects $\left(F_{(2,18)}=0.21 ; p=0.813\right)$. The clustering results were replicated using a $k$-means analysis with $k=3$ (mutual information $[\mathrm{MI}]=0.853$; Fig. $2 a$ ) and also stable over a larger range of $k(2-20 ; \mathrm{MI}>0.4)$.

Nonparametric permutation testing. To determine the network signature of each cluster, we compared the frequency of the network identity of each stimulus-recording pair to a permutation analysis (5000 iterations) and then estimated the frequency of each network pair in each cluster. Data were initially randomized within subject before recombination at the group level to ensure that individual subject data were not responsible for any group-level effects. Pairs more extreme than the highest $1 \%$ were taken to be significant (Nichols and Holmes, 2002). Importantly, this approach does not confirm the presence or absence of a particular intranetwork or internetwork connection within an individual cluster per se, but rather identifies the directional connections that were present in each cluster above chance levels.

This analysis was run three separate times: the first two analyses occurred at the network level, first independently of which other network was activated and subsequently at the level of internetwork interactions. These results are depicted in Figure $2 c$ : intranetwork and internetwork connections that were more prevalent in each cluster than the most extreme values found in the permutation analysis are represented by thick black arrows within and between each of the three networks. Similar analyses were then conducted at the level of interregional interactions. Figure $2 d$ shows the connections between networks that were either associated with significant elevated evoked activity, however, in this case, thick black lines represent strong interregional connections $(p<0.001)$, whereas thin black lines represent weaker connections $(p<0.05)$. Nonsignificant results are shown in gray.

Post hoc analyses. To confirm independently the results of the clustering analysis, we performed a series of post hoc analyses. First, we calculated the grand mean of each time series after they were organized according to the network that was stimulated. Time series data were then binned into the activation that occurred before the end of the first cluster (i.e., before $t=+70 \mathrm{~ms}$ ) or after the start of the second cluster (i.e., after $t=+82 \mathrm{~ms}$ ). This step was used in an attempt to differentiate "early" (i.e., cluster 1) and "late" (i.e., cluster 2 and 3) activation patterns, but care was taken to ensure that results were similar when each of the latter
Table 2. Table of pairs of electrodes stimulated and recorded from across the three network categories

\begin{tabular}{llrl}
\hline & Recorded & & \\
\cline { 2 - 4 } & SN & FPN & DN \\
\hline Stimulated & & & \\
SN & 33 & 91 & 196 \\
FPN & 21 & 278 \\
DN & 105 & 225 \\
\hline
\end{tabular}

DN, default network; FPN, frontoparietal network; SN, salience network.

clusters was analyzed separately. The mean $Z$-score within each bin was then compared at the group level using a $2 \times 2$ ANOVA. We further confirmed the general pattern observed in this analysis at the individual subject level by calculating a difference score between the early and late bins for each group of regions. Five of seven subjects demonstrated a relationship commensurate with the group-level results. This result was then compared with a null distribution in which we shuffled the labels of each value 1000 times and then calculated the proportion of subjects with similar patterns $\left(95^{\text {th }}\right.$ percentile of permuted data $\left.=3 / 7\right)$. Our final two post hoc analyses both used the same approach as the first post hoc analysis except that they focused on different relationships: the first contrasted the bidirectional relationship between DN and FPN, whereas the final post hoc analysis compared DN activity after stimulation of either DN or FPN/SN. Due to the nonparametric nature of the stimulated time series, we used Mann-Whitney $U$ tests to determine differences in activity after stimulation in each analysis.

Euclidean distance between significant pairs of electrodes. As a final experiment, we calculated the Euclidean distance between each pair of electrodes (both stimulated and recorded from) across the cohort of seven subjects. As a first step, we correlated the time to peak onset after stimulation to the Euclidean distance between the stimulated and recorded electrode pairs using a Spearman's $\rho$ correlations within each subject. We next investigated whether the Euclidean distance between electrode pairs (which itself is a proxy measure of the relative distance of propagation between two regions) differed as a function of the network pairs that were significantly associated with activity in either the early cluster $(\mathrm{SN} \rightarrow \mathrm{SN}$; FPN $\rightarrow \mathrm{SN}$ ), middle cluster $(\mathrm{SN} \rightarrow \mathrm{FPN})$, or late cluster $(\mathrm{DN} \rightarrow \mathrm{DN} ; \mathrm{DN} \rightarrow \mathrm{SN})$. DN $\rightarrow$ FPN connections that were significantly present in both the middle and late cluster were discarded. A set of nonparametric, Mann-Whitney $U$ tests was used to compare the Euclidean distance across the three clusters.

Data and software availability. Further information and requests for data sharing and code may be directed to and will be fulfilled by the corresponding author.

\section{Results}

Data-driven clustering of stimulation evoked responses

Single pulses were delivered within each of the pre-identified nodes of the 3 networks (36 sites; 40 single square-wave biphasic pulses in each site, one pulse every 2 s; Fig. 1, Table 2). During the stimulations, we recorded cortico-cortico-evoked potentials in the $250 \mathrm{~ms}$ window after electrical stimulation (Matsumoto et al., 2004). In total, we recorded from 220 cortical sites across seven subjects. Our final sample thus yielded 987 unique paired time series, each of which corresponded to two pairs of electrodes: one pair that was stimulated and one that was recorded from (Fig. 1, Table 2). Across subjects, there was a positive relationship between the Euclidean distance between electrode pairs and the time to peak onset (mean $\rho=0.341 \pm 0.14$ ).

Our analysis revealed marked heterogeneity in temporal activity across stimulation and recording sites (Fig. $2 a, b$ ). To provide a readily interpretable summary of these data, we performed a clustering analysis, which clearly identified three spatiotemporally distinct clusters (Fig. 2c,d); these were confirmed using additional clustering techniques (see Materials and Methods). 
Visualization of the mean activity within each cluster demonstrated three distinct temporal patterns (Fig. 2c). The first cluster (Fig. $2 c, d$, orange) demonstrated significantly elevated activity within an early window (significantly elevated activity between +11-69 ms after stimulation), likely the initial, feedforward, excitatory response to stimulation (Keller et al., 2014). The second (+82-125 ms; Fig. $2 c, d$, pink) and third clusters $(+132-200$ ms; Fig. $2 c, d$, purple) peaked within later windows, likely reflecting activity that propagated along multisynaptic pathways (Maris and Oostenveld, 2007; Borchers et al., 2011; Keller et al., 2014).

\section{Network signature of stimulation patterns}

Using the clustering assignments identified in the previous step, we next used a nonparametric permutation approach to determine the presence of particular network (Fig. 3a) and regional (Fig. 3b) pairs that were present in each of the three clusters above chance levels $(p<0.05)$. The results of the permutation analysis presented a clear pattern (Fig. $3 a$ ): the first cluster was associated with stimulation of the FPN and SN (but not DN) that evoked fast responses within the SN. The second cluster was associated with a significant number of pairs of electrodes in which stimulation of the $\mathrm{SN}$ and DN evoked activity within the frontal regions of the FPN. The final cluster was characterized by stimulation of regions within the $\mathrm{DN}$, which demonstrated a relatively delayed activation of sites within the DN or frontal regions within the FPN and SN. It should be noted that there were no significant differences in the Euclidean distance between the network pairs identified with each cluster (mean Euclidean distance between stimulated and recorded pairs in cluster 1: $64.99 \pm 28.05$; cluster $2: 62.62 \pm 26.79$; cluster $3: 64.74 \pm 28.46 ; p>0.500$ ), suggesting that the approximate synaptic distance between regions was not an effective explanation for the heterogeneity of our results.

Each of the network-level interactions was also associated with specific regional substrates (Fig. $3 b ; p<0.001$ ). Specifically, the SN evoked activity identified in the first cluster (+11-69 ms) was predominantly driven by temporoparietal junction (TPJ)mediated activation of anterior medial cingulate cortex (aMCC), along with intraregional activity within the SN. The FPN evoked activity observed in the first cluster was observed above chance in prefrontal anterior cingulate cortex (pACC) and posterior parietal cortex (PCC), with both regions activating the aMCC, but only the PCC activating the TPJ above chance. The second cluster, which peaked between $+82-125 \mathrm{~ms}$, was characterized by dorsolateral prefrontal cortex evoked activity from stimulation of the anterior insula/frontal operculum (AI/fO) and pACC evoked activity from stimulation of posteriomedial cortex (PMC). The third cluster (+132-200 ms) was associated with medial temporal lobe (MTL)-driven activation of the aMCC and PMC-mediated activation of $\mathrm{AI} / \mathrm{fO}$ and MTL. There were other patterns observed at the regional level at lower levels of statistical significance (Fig. $3 c$, thin lines).

A significant proportion of stimulated DN regions existed within the MTL, which, although classically associated with the $\mathrm{DN}$, contains a relatively unique connectivity profile (van den Heuvel et al., 2015). Therefore, it is possible that some of our results may have been driven in part by factors unique to regions within the MTL. Overall, the effect of stimulating MTL nodes was similar to other DN regions, with the majority of significant responses occurring in the latter two clusters from Figure $2 b$. However, when decomposing the DN into MTL-related and MTL-unrelated regions, we found that non-MTL regions were predominantly responsible for the internetwork effects observed in the second cluster (i.e., DN $\rightarrow$ FPN), whereas both MTL and $\mathrm{DN}$ regions were actively responsible for internetwork effects observed in the third cluster (i.e., DN/MTL $\rightarrow$ other networks). These results suggest a relatively late effect (i.e., in the third temporal cluster; +132-200 ms after stimulation) for regions in the MTL subdivision of the DN.

\section{Post hoc analyses}

To confirm that the results of our experiment were not due to idiosyncrasies associated with the clustering approach, we performed a series of additional analyses. We first confirmed the finding that stimulation of the FPN or SN led to higher activation 
at the early window, whereas stimulation of the DN led to relatively higher activations in the delayed window. In doing so, we found that the magnitude of evoked responses across all recorded sites was clearly maximal in the earlier (i.e., $<70 \mathrm{~ms}$ ) rather than later windows when the FPN was stimulated, whereas DN stimulation caused maximal responses in the later windows (significant interaction effect: $p=10^{-4}$ ) and stimulation of the SN was associated with a relative balance between the two windows. In addition, we were also able to partially replicate the finding that intranetwork stimulation amplitudes were greater than internetwork effects (Keller et al., 2014), a pattern that held for both DN $\left(Z=5.69 ; p=10^{-8}\right)$ and $\operatorname{FPN}\left(Z=6.53 ; p=10^{-11}\right)$, but not SN, where activation was more equivocal across the two categories $(Z=0.42 ; p=0.627)$.

To rule out the possibility that our findings were due to the influence of idiosyncratic patterns across individual subjects, we next looked at the presence of temporal network connectivity at the individual level. There are a number of reasons to expect that patterns within individual subjects may differ. First the electrode location for each of the intrinsic networks varied slightly from one subject to another (Table 1 ). Second, a relatively short restingstate session was used to identify network location, which may have biased toward deviations from "stable" network architecture in individual subjects (Laumann et al., 2015). Finally, the registration between MRI and CT can also be problematic given issues associated with perisurgical edema. Despite these potential sources of errors, we found that late responses were predominant in the DN in all seven subjects, whereas early responses were present within the FPN and SN in five of seven subjects (i.e., $71 \%$ ). Importantly, a permutation test demonstrated that this value was significant greater than chance $\left(95^{\text {th }}\right.$ percentile of permutation test $=42.9 \%$ ), suggesting that the observed group-level relationship was reasonably robust at the single-subject level.

To verify interregional patterns observed in the clustering analysis, we confirmed that the DN activated FPN selectively in later windows (Fig. $4 a, b ; Z=3.03 ; p=0.002$ ), whereas the FPN had relatively little effect on the DN sites in either the early or the late window $(Z=-0.91 ; p=0.365)$. The interaction between these two patterns was also significant $\left(p=10^{-10}\right)$. Furthermore, we showed that the $\mathrm{DN}$ received poststimulation activity preferentially after DN stimulation compared with stimulation of either FPN or SN. Specifically, the mean $Z$-score across the entire poststimulation time series $(+10$ to $+200 \mathrm{~ms}$ ) in the DN after self-stimulation (normalized amplitude $=2.36 \pm 0.53$ ) was significantly greater than the activity after FPN or SN stimulation (normalized amplitude $=1.65 \pm 0.41 ; Z=12.4 ; p=10^{-35}$ ). Together, these results confirm our data-driven approach and highlight the asymmetrical and temporal specific patterns of connectivity present across the three networks.

\section{Discussion}

Our findings reveal crucial information for understanding coordinated activity between the large-scale networks of the human brain and suggest important heterogeneity in pathways of internetwork communication. The evoked responses between networks demonstrated distinct temporal patterns of signal propagation (Fig. 3 ) because stimulation of the FPN caused higher modulations at earlier stages of processing, whereas the DN demonstrated a greater and sustained influence on the FPN and SN at a relatively later stage. These findings clearly suggest that selective patterns of putative signal propagation occur within distinct directions and according to distinct temporal scales.
In particular, our data provide novel information that is relevant to models of dynamic internetwork interactions, particularly as they relate to switching between networks and the relative temporal receptive windows that relate to optimal network functionality (Honey et al., 2012). For instance, a growing body of evidence suggests that the brain displays a temporal hierarchy that may relate to heterogeneous patterns of interregional structural connectivity (Chaudhuri et al., 2015; Mitra and Raichle, 2016). Alternatively, the low-frequency signatures of particular regions might interact to create qualitatively distinct patterns as neural regions become further isolated from peripheral sensory and motor constraints (Baria et al., 2013). On the surface, our data appear to be consistent with both models (Stephens et al., 2013) and further suggest that local computational capacities and inter-areal heterogeneity within the FPN, SN, and DN regions may explain the observed differences in the temporal scales of effective connectivity among the three networks.

Our empirical confirmation of these models sets the stage for testing clear hypotheses in the future: for instance, it could be hypothesized that the DN influences the working of SN and FPN (as well as other nodes within the DN) after they have completed their (early) local processing, perhaps reflecting a delay in the temporal influence of the DN that might expedite the integration of the products of their local information processing over time (Hasson et al., 2015). Other studies could also determine whether these same patterns of effective connectivity are modulated by task demands that recruit activity within each of the networks investigated in this study. Finally, the finding that MTL-related regions of the DN were involved in the latter clusters suggests a potential temporal signature of memory-related processing, a hypothesis that requires further elaboration in targeted functional studies.

Overall, our study represents an important step toward mapping specific patterns of information flow around the brain and thus provides a framework for studying the structure of networklevel dynamics within the brain over time. In doing so, our results provide insights into the spatiotemporal dynamics that are likely to constrain the architecture of the brain networks supporting human cognition and behavior.

\section{References}

Baria AT, Mansour A, Huang L, Baliki MN, Cecchi GA, Mesulam MM, Apkarian AV (2013) Linking human brain local activity fluctuations to structural and functional network architectures. Neuroimage 73:144155. CrossRef Medline

Borchers S, Himmelbach M, Logothetis N, Karnath HO (2012) Direct electrical stimulation of human cortex: the gold standard for mapping brain functions? Nat Rev Neurosci 13:63-70. CrossRef Medline

Buckner RL, Andrews-Hanna JR, Schacter DL (2008) The brain's default network: anatomy, function, and relevance to disease. Ann N Y Acad Sci 1124:1-38. CrossRef Medline

Buckner RL, Krienen FM, Yeo BT (2013) Opportunities and limitations of intrinsic functional connectivity MRI. Nat Neurosci 16:832-837. CrossRef Medline

Chaudhuri R, Knoblauch K, Gariel MA, Kennedy H, Wang XJ (2015) A large-scale circuit mechanism for hierarchical dynamical processing in the primate cortex. Neuron 88:419-431. CrossRef Medline

Corbetta M, Shulman GL (2002) Control of goal-directed and stimulusdriven attention in the brain. Nat Rev Neurosci 3:215-229. CrossRef Medline

Glover GH, Law CS (2001) Spiral-in/out BOLD fMRI for increased SNR and reduced susceptibility artifacts. Magn Reson Med 46:515-522. Medline

Gollo LL, Roberts JA, Cocchi L (2017) Mapping how local perturbations influence systems-level brain dynamics. Neuroimage. Advance online publication. Retrieved September 12, 2017. doi: 10.1016/j.neuroimage. 2017.01.057. 
Gordon EM, Laumann TO, Gilmore AW, Newbold DJ, Greene DJ, Berg JJ, Ortega M, Hoyt-Drazen C, Gratton C, Sun H, Hampton JM, Coalson RS, Nguyen AL, McDermott KB, Shimony JS, Snyder AZ, Schlaggar BL, Petersen SE, Nelson SM, Dosenbach NUF (2017) Precision functional mapping of individual human brains. Neuron 95:791-807. Medline

Hasson U, Chen J, Honey CJ (2015) Hierarchical process memory: memory as an integral component of information processing. Trends Cogn Sci 19:304-313. CrossRef Medline

Honey CJ, Thesen T, Donner TH, Silbert LJ, Carlson CE, Devinsky O, Doyle WK, Rubin N, Heeger DJ, Hasson U (2012) Slow cortical dynamics and the accumulation of information over long timescales. Neuron 76:423434. CrossRef Medline

Keller CJ, Honey CJ, Mégevand P (2014) Mapping human brain networks with cortico-cortical evoked potentials. Philos Trans R Soc Lond B Biol Sci 369: pii: 20130528. CrossRef Medline

Laumann TO, Gordon EM, Adeyemo B, Snyder AZ, Joo SJ, Chen MY, Gilmore AW, McDermott KB, Nelson SM, Dosenbach NU, Schlaggar BL, Mumford JA, Poldrack RA, Petersen SE (2015) Functional system and areal organization of a highly sampled individual human brain. Neuron 87:657-670. CrossRef Medline

Maris E, Oostenveld R (2007) Nonparametric statistical testing of EEG-and MEG-data. J Neurosci Methods 164:177-190. CrossRef Medline

Matsumoto R, Nair DR, LaPresto E, Najm I, Bingaman W, Shibasaki H, Lüders HO (2004) Functional connectivity in the human language system: a cortico-cortical evoked potential study. Brain 127:2316-2330. CrossRef Medline

Mitra A, Raichle ME (2016) How networks communicate: propagation patterns in spontaneous brain activity. Philos Trans R Soc Lond B Biol Sci 371: pii: 20150546. CrossRef Medline

Nichols TE, Holmes AP (2002) Nonparametric permutation tests for func- tional neuroimaging: a primer with examples. Hum Brain Mapp 15:1-25. CrossRef Medline

Raichle ME (2015) The brain's default mode network. Annu Rev Neurosci 38:433-447. CrossRef Medline

Ramsey JD, Hanson SJ, Hanson C, Halchenko YO, Poldrack RA, Glymour C (2010) Six problems for causal inference from fMRI. Neuroimage 49: 1545-1558. CrossRef Medline

Rubinov M, Sporns O (2010) Complex network measures of brain connectivity: uses and interpretations. Neuroimage 52:1059-1069. CrossRef Medline

Seeley WW, Menon V, Schatzberg AF, Keller J, Glover GH, Kenna H, Reiss AL, Greicius MD (2007) Dissociable intrinsic connectivity networks for salience processing and executive control. J Neurosci 27:2349-2356. CrossRef Medline

Stephens GJ, Honey CJ, Hasson U (2013) A place for time: the spatiotemporal structure of neural dynamics during natural audition. J Neurophysiol 110:2019-2026. CrossRef Medline

Uddin LQ (2015) Salience processing and insular cortical function and dysfunction. Nat Rev Neurosci 16:55-61. CrossRef Medline

van den Heuvel MP, Scholtens LH, Feldman Barrett L, Hilgetag CC, de Reus MA (2015) Bridging cytoarchitectonics and connectomics in human cerebral cortex. J Neurosci 35:13943-13948. CrossRef Medline

Wang D, Buckner RL, Fox MD, Holt DJ, Holmes AJ, Stoecklein S, Langs G, Pan R, Qian T, Li K, Baker JT, Stufflebeam SM, Wang K, Wang X, Hong B, Liu H (2015) Parcellating cortical functional networks in individuals. Nat Neurosci 18:1853-1860. CrossRef Medline

Yeo BT, Krienen FM, Sepulcre J, Sabuncu MR, Lashkari D, Hollinshead M, Roffman JL, Smoller JW, Zöllei L, Polimeni JR, Fischl B, Liu H, Buckner RL (2011) The organization of the human cerebral cortex estimated by intrinsic functional connectivity. J Neurophysiol 106:1125-1165. CrossRef Medline 